\title{
PDE-4-Inhibitor in GOLD-Leitlinien aufgenommen
}

_ In den Ende 2010 aktualisierten Leitliniene der Global Initiative for Chronic Obstructive Lung Disease (GOLD) wurde Roflumilast (Daxas ${ }^{\oplus}$ ) als neue Therapieoption für die Behandlung der COPD aufgenommen. Die "Global Strategie für Diagnose, Behandlung und Prävention von COPD" enthält evidenzbasierte Leitlinien für die Behandlung von

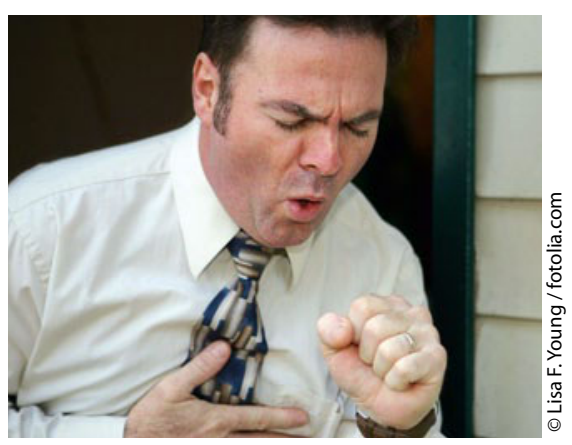

Die antiinflammatorische PDE-4-Therapie reduziert COPD-Exazerbationen.
COPD und wird jährlich von einem Gremium führender COPD-Experten aktualisiert. Die aktuelle Ausgabe fasst im Abschnitt zu pharmakologischen Therapien auch die Charakteristika des Phosphodiesterase-4-(PDE4-) Inhibitoren Roflumilast bei COPD-Patienten zusammen. Die seit 2010 in Deutschland bei schwerer COPD zugelassene Substanz wirkt gezielt über die Hemmung der inflammatorischen Komponente der Erkrankung.

\section{Entzündung verringern, Häufigkeit von Exazerbationen senken}

Die GOLD-Leitlinien bestätigen, dass die PDE-4-Inhibitoren in erster Linie die der COPD zugrundeliegenden Entzündungen und deren klinische Folgen verringern. Roflumilast reduzierte in klinischen Studien die Häufigkeit von Exazerbationen bei Patienten mit schwerer oder sehr schwerer COPD (Stadien III-IV) und chronischer Bronchitis sowie häufigen Exazerbationen [1].
„COPD ist trotz der schon bestehenden Therapien nach wie vor eine Erkrankung mit einem großen medizinischen Bedarf", betonte Prof. Peter Calverley, Lungenfacharzt aus Liverpool und Exekutivmitglied der GOLD-Initiative [2]. „Häufig leiden die Patienten an Exazerbationen, bei denen die Atmung äußerst stark beeinträchtigt sein kann und die Patienten oft stationär behandelt werden müssen. Studien haben gezeigt, dass der PDE-4-Inhibitor Roflumilast Exazerbationen signifikant reduziert. Mit der Aktualisierung erkennen die GOLD-Leitlinien die Bedeutung dieser neuen Behandlungsoption bei COPD und die Notwendigkeit an, die Ärzte darüber zu informieren", resümierte Calverley.

$\operatorname{tr}$

Quellen: 1.The Global Strategy for Diagnosis, Management and Prevention of COPD (Update 2010): http://www.goldcopd.com 2. Pressemitteilung Nycomed vom 7.01.2011

\section{Spezifische Immuntherapie mit Evidenz}

\section{Sublingualtablette erfolgreich bei allergischer Rhinitis}

— Für die spezifische Immuntherapie (SIT) bei allergischen Erkrankungen gab es bis vor kurzem wenig klinische Evidenz. Zwar belegten kontrollierte klinische Studien die Wirksamkeit der SIT, hinsichtlich Qualität und Quantität genügten sie modernen Maßstäben jedoch nicht. Entsprechend unübersichtlich war die Marktsituation mit mehr als 6500 Einzelpräparaten, ungeprüften Mischungen und unterschiedlichsten Dosierungen, die zum Teil die Zulassungsbestimmungen als so genannte Individualrezepturen umgingen.

Erst mit den Richtlinien der European Medicines Agency (EMA) zur klinischen Entwicklung von Therapieallergenen sei die SIT in der evidenzbasierten Medizin angekommen, erklärte Prof. Ulrich Wahn, Berlin: „Die Immuntherapie muss heute die gleiche Messlatte nehmen wie die Pharmakotherapie." Voraussetzung für die Zulassung sind demnach randomisierte placebokontrollierte doppelblinde Multizenterstudien in adäquater Größe und mit ausreichenden Patientenzahlen. Gefordert werden Untersuchungen zur Pharmakodynamik und Dosisfindung, zur Kurz- und Langzeitwirksamkeit und Sicherheit mit validen Endpunkten. Eine Marktbereinigung erwartet Prof. Stefan Vieths, Langen, von der Therapieallergene-Verordnung: Danach dürfen alle Präparate, für die bis Ende 2010 keine Zulassung beantragt wurde, nicht mehr verkauft werden.

\section{Hohe Symptomreduktion schon nach einem Jahr}

Die 5-Gräser-Tablette Oralair ${ }^{\circledast}$ erfüllt bereits diese Forderungen. Die Sublingualtablette ist zugelassen für die SIT bei gräserpollenbedingter allergischer Rhinitis bei Erwachsenen sowie bei Kindern ab fünf Jahren. In umfangreichen Studienprogrammen geprüft, zeigte die sublinguale Immuntherapie (SLIT) eine Symptomreduktion um 39\% bei Kindern und $37 \%$ bei Erwachsenen im ersten Jahr der Behandlung. Im dritten Jahr war der Symptomscore um $50 \%$ reduziert. Als optimale Dosis erwiesen sich 300 IR, berichtete Prof. Randolf Brehler aus Münster: „Eine weitere Dosisstei- gerung brachte keinen zusätzlichen Effekt mehr." Bereits vier Wochen nach Therapiebeginn zeigt sich ein signifikant besserer Effekt als unter Placebo, am Studienende nach 120 Tagen war der Unterschied 29,3\%. Empfohlen wird ein Therapiebeginn vier Monate präsaisonal mit kosaisonaler Fortsetzung.

\section{Wirksamkeit in der Praxis bestätigt}

Dr. Ulrich Ecke, Frankfurt, bestätigte diese Daten mit Erfahrungen aus der eigenen Praxis. Die hohe Erfolgsquote mit Symptomreduktion um bis zu 50\%, verringerter Symptomatikaeinnahme und gesteigerter Lebensqualität machten die SLIT zur gleichwertigen Alternative zur subkutane Immuntherapie. Studien zeigten vergleichbare Effektivität der beiden Applikationsformen. Wegen der geringeren Invasivität und Nebenwirkungsrate ist die SLIT für Ecke Mittel der Wahl.

koc

Quelle: Expert-Meeting „Die Dimension der evidenzbasierten SIT" am 28. Januar 2011 in Berlin (Veranstalter: Stallergenes) 